\title{
MULTI-APERTURE FOCUSING IN SPACEBORNE TRANSMITTER-STATIONARY RECEIVER BISTATIC SAR
}

\author{
Andrei Anghel ${ }^{\star} \quad$ Remus Cacoveanu ${ }^{\S \star} \quad$ Bjorn Rommen* Mihai Datcu ${ }^{\star \ddagger}$ \\ * University POLITEHNICA of Bucharest, CEOSpaceTech, Bucharest, Romania \\ $\S$ EOS Electronic Systems, Bucharest, Romania \\ ${ }^{*}$ European Space Agency (ESA-ESTEC), Noordwijk, The Netherlands \\ $\ddagger$ German Aerospace Center (DLR), Oberpfaffenhofen, Germany
}

\begin{abstract}
The paper proposes a methodology to perform azimuth focusing of spaceborne transmitter-stationary receiver bistatic synthetic aperture radar (SAR) data across multiple alongtrack apertures to increase azimuth resolution. The procedure uses as input several azimuth apertures (continuous groups of range compressed pulses) from one or more satellite bursts and comprises the following stages: azimuth antenna pattern compensation, slow time resampling, reconstruction of missing azimuth samples between neighbouring sets of pulses using an auto-regressive model and back-projection focusing of the resulting multi-aperture range image. The approach is evaluated with real bistatic data acquired over an area of Bucharest city, Romania.
\end{abstract}

Index Terms - Bistatic SAR, Focusing, Signal reconstruction.

\section{INTRODUCTION}

A certain location from the ground can be usually imaged with a monostatic spaceborne synthetic aperture radar (SAR) sensor by exploiting the information available from one ascending/descending orbit. Moreover, if the operating mode of the satellite uses multiple sub-swaths this information is obtained only from one sub-swath per orbit. Bistatic SAR imaging systems with a stationary receiver and a spaceborne transmitter of opportunity (e.g., TerraSAR-X [1], ERS2/ENVISAT [2] or GNSS [3]) open the possibility to image the same area using data bursts belonging to multiple subswaths $[4,5]$.

When Sentinel-1A/B operating in the terrain observation through progressive scans (TOPSAR) imaging mode is used as transmitter of opportunity, a stationary receiver captures pulses from the burst corresponding to the sub-swath in which the receiver is placed, and also from bursts belonging to the other sub-swaths. In both cases, the received pulses can be pulses that were transmitted through the main beam or through the side lobes of the satellite's antenna. The available multi-burst data can be used in various ways for target characterization by exploiting the enhanced azimuth diversity.
A procedure to enhance the azimuth resolution using pulses received through the antenna's sidelobes was presented in [4] for a bistatic receiver that records data continuously.

This paper presents a methodology developed to increase azimuth resolution by exploiting multi-aperture bistatic data acquired in a spaceborne transmitter-stationary receiver configuration. The procedure uses as input several continuous groups of range compressed pulses (from one or more bursts) and consists in the following main steps: compensation of the azimuth antenna pattern (AAP), resampling in the slow time domain and reconstruction of the missing azimuth samples between neighbouring groups of pulses using an autoregressive (AR) model. The obtained multi-aperture range image is focused on a two-dimensional grid with a backprojection algorithm adapted to bistatic geometry.

Compared to the method presented in [4], the procedure proposed in this paper aims to exploit data from multiple bursts and is designed for a trigger-based acquisition, which reduces the memory requirements and enhances the maximum number of pulses that can be recorded. This implies an increased total aperture length (and better azimuth resolution), but the pulses that do not reach the triggering threshold will not be recorded and their information has to be extrapolated from available data.

The rest of this paper is structured as follows. Section II presents the developed procedure to focus multi-aperture bistatic data, while in Section III the methodology is evaluated with real data acquired with the receiver presented in [5]. The conclusions are stated in Section IV.

\section{MULTI-APERTURE FOCUSING OF BISTATIC DATA}

\subsection{Bistatic azimuth signal model}

The envisaged bistatic geometry is shown schematically in Fig. 1. The ground receiver has two channels -one that receives directly the transmitted pulses through an antenna oriented towards the satellite (reference channel) and another that receives the reflected signals from the illuminated scene (typically called imaging channel). The duration of each aper- 


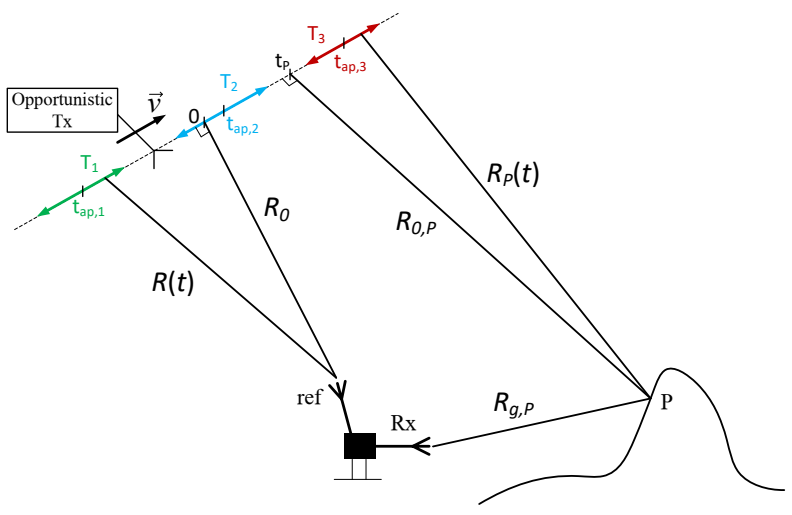

Fig. 1. Spaceborne transmitter-stationary receiver bistatic geometry.

ture $k$ that illuminates the scene is denoted with $T_{k}$ and its central azimuth time with $t_{a p, k}$. The closest approach distances to the receiver and to a point $\mathrm{P}$ from the scene are $R_{0}$, and respectively, $R_{0, P}$. The origin of the slow time axis corresponds to the closest point of approach to the receiver and $t_{P}$ is the zero-Doppler time of point $\mathrm{P}$. At the ground receiver level, the range compression of each pulse is performed by inter-correlating the baseband signal of the imaging channel with the one of the reference channel [5], and the azimuth bistatic signal of point $\mathrm{P}$ seen from aperture $k$ can be expressed as:

$$
\begin{aligned}
& s_{k}(t)=A \exp \left[-j \frac{2 \pi}{\lambda}\left(\sqrt{R_{0, P}{ }^{2}+\left[v\left(t-t_{P}\right)\right]^{2}}+R_{g, P}\right)\right] \times \\
& \times \exp \left(j \frac{2 \pi}{\lambda} \sqrt{R_{0}^{2}+(v t)^{2}}\right) p_{k}(t) \approx A \exp \left(-j \frac{2 \pi}{\lambda} R_{g, P}\right) \times \\
& \times \exp \left\{j \frac{2 \pi}{\lambda}\left[\left(R_{0}-R_{0, P}\right)-\frac{v^{2} t_{P}^{2}}{2 R_{0, P}}+\frac{v^{2} t_{P}}{R_{0, P}} t\right]\right\} p_{k}(t) \times \\
& \times \exp \left\{j \frac{2 \pi}{\lambda}\left[\frac{v^{2}}{2}\left(\frac{1}{R_{0}}-\frac{1}{R_{0, P}}\right) t^{2}\right]\right\}
\end{aligned}
$$

where $A$ is the complex amplitude, $\lambda$ is the wavelength at central frequency, $v$ is the satellite's velocity and $p_{k}(t)$ is a window centered around $t_{a p, k}$, which includes the effect of the AAP. Note that $s_{k}(t)$ is a chirp signal in the slow time domain with a small chirp rate (for a target placed at $R_{g, P}=10 \mathrm{~km}$ from the ground receiver and Sentinel-1 used as transmitter of opportunity, the bistatic chirp rate is around $30 \mathrm{~Hz} / \mathrm{s}$, which is a few hundred times smaller than the monostatic one), so the bistatic azimuth signal actually consists in complex exponentials with slowly varying frequency (the inter-correlation with the reference channel signal acts almost like a de-chirping in azimuth). From the implementation point of view, the sampled version of the signal $s_{k}(t)$ is a group of $N_{p, k}$ pulses acquired with a certain pulse repetition interval (PRI).

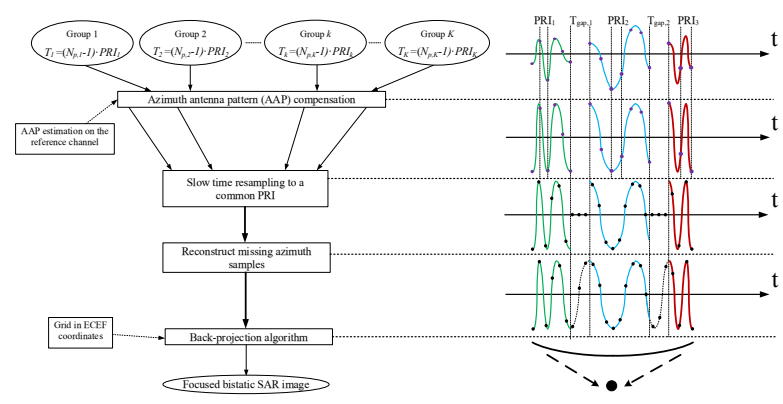

Fig. 2. Multi-aperture bistatic focusing: block diagram and qualitative representation of intermediate signals.

\subsection{Bistatic azimuth focusing}

A block diagram of the procedure used to focus bistatic data from multiple apertures is presented in Fig. 2. We consider $K$ groups of consecutive pulses separated by gaps of different durations. The duration of the gap between group $k$ and group $k+1$ is denoted with $T_{g a p, k}$. The PRI of each group depends on the burst to which it belongs (usually we have a few consecutive groups with the same PRI placed between groups with different PRIs).

Due to the beam sweep in a TOPSAR mode, each aperture (group of pulses) will be affected in a different way by the AAP. Since the receive angle of the reference channel antenna is practically constant in the entire aperture, the actual AAP for each group of pulses is proportional to the amplitude of the pulses received on the reference channel. Therefore the normalized AAP can be estimated on the reference channel data and the compensation can be performed by dividing the imaging data with the AAP. In [4] the AAP compensation is done with a weighing window depending on the signal-to-noise ratio (SNR) in order to reduce noise amplification (however, for large SNRs the weighing window is practically the inverse of the AAP).

Since the apertures can be sampled with different PRIs, all range compressed data are resampled in slow time domain to a common PRI. If the BP focusing algorithm is applied directly on the azimuth phase history with inter-aperture gaps (considering as zeros the azimuth samples in between the apertures), grating lobes will appear in the final bistatic SAR image. To avoid this effect, the next step is to fill the gaps between the apertures using an AR model [6].

Restoration of lost samples using AR models is typically used for speech and music signals, and the technique is capable to reconstruct the signal in gaps of up to $100 \mathrm{~ms}[6,7]$. This method can be used to fill the inter-aperture gaps of bistatic azimuth signals since they actually consist in a couple of linear chirp signals with small frequency variations (quite similar with some audio signals) that can easily be regarded as short-term stationary signals around the gaps. 

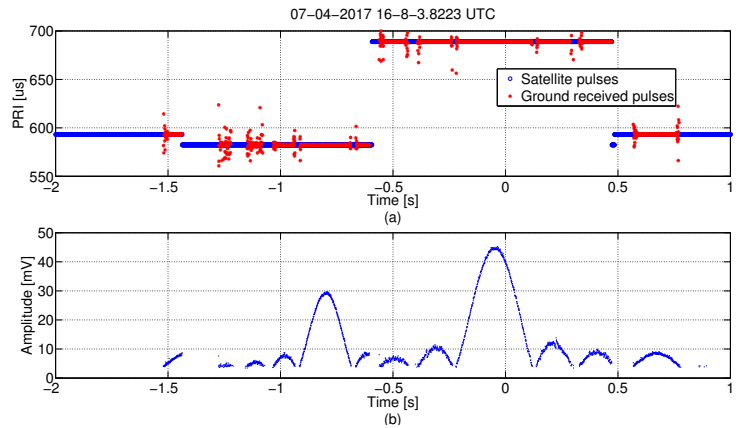

Fig. 3. Received pulses for the acquisition performed on 07.04.2017: a) PRI (nominal and computed from the timestamps) and b) amplitude.

In our implementation, the samples of a gap are determined as an average of the signals predicted by two ARmodels: one estimated using the samples from the left-side of the gap (forward prediction) and another based on the samples from the right-side of the gap (backward prediction). This approach lessens the short-term stationarity requirements imposed to the signal [6]. The order of the model is chosen such that it minimizes the Akaike information criterion over the remaining samples [8].

\section{EXPERIMENTAL EVALUATION}

This section presents an evaluation of the proposed multiaperture focusing procedure with a bistatic acquisition performed on 07.04.2017 over an area of Bucharest city, Romania, with the ground receiver being placed on top of the rectorate building of University Politehnica of Bucharest and the Sentinel-1B satellite flying on an ascending orbit. The implementation details of the ground receiver are presented in [5]. The PRI and amplitude of the received pulses versus slow time are shown in Fig. 3. Fig. 3(a) displays the nominal PRI for each sub-swath and the PRI computed as difference between consecutive GPS timestamps stored by the ground receiver, whereas Fig. 3(b) shows the amplitude of each received pulse. The maximum amplitude is obtained for the burst from sub-swath 2 (with 689 us PRI), but there are also pulses received from bursts belonging to sub-swaths 1 and 3 .

\subsection{Signal reconstruction assessment}

In this subsection we assess the impact of the AR-based signal reconstruction on the focused image. In this regard, we consider only the main beam of the azimuth signal (between -0.21 $\mathrm{s}$ and $0.11 \mathrm{~s}$ slow time) and artificially introduce gaps of different sizes in the middle of the lobe. The multi-aperture focusing procedure is applied on the resulting signal (two apertures with one continuous gap in between) with and without the signal reconstruction stage. The focused bistatic images

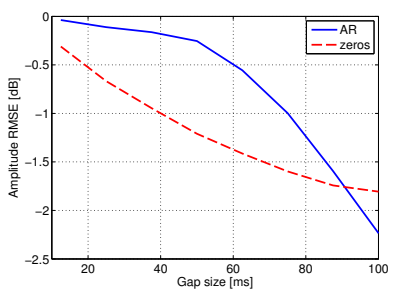

(a)

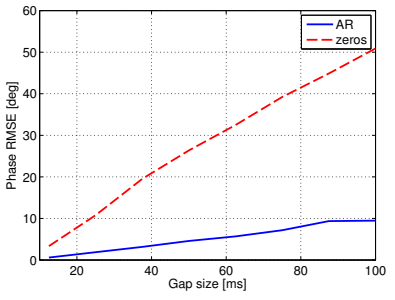

(b)
Fig. 5. Comparison between multi-aperture focusing with/without azimuth signal reconstruction and the reference image: RMSE of (a) amplitude and (b) phase.

are compared with the reference one obtained without any gap (classical focusing). The grid used in the BP algorithm is aligned with the latitude/longitude axis and its origin is the receiver's position.

Fig. 4(a) presents the reference image, Fig. 4(b) displays the focused image with zero-filled gaps, while Fig. 4(c) shows the focused image after AR model-based gap filling for a $50 \mathrm{~ms}$ gap duration. In Fig. 5 we present the root mean squared error (RMSE) of the amplitude and phase of the targets with normalized amplitude (to the most reflective target in the scene) above $-20 \mathrm{~dB}$. Note that without azimuth signal reconstruction there are several grating lobes that affect the image and the phase is quite biased. After gap filling the obtained image resembles the reference one, with the exception of a few sidelobes. Additionally, the phase error of highly reflective targets for a gap duration below $0.1 \mathrm{~s}$ is around a few degrees.

\subsection{Resolution enhancement}

To emphasis the azimuth resolution enhancement that can be obtained by multi-aperture focusing we applied the procedure on the azimuth signal shown in Fig. 3 for the groups of consecutive pulses between $-1.02 \mathrm{~s}$ to $0.76 \mathrm{~s}$. In this interval, the maximum gap size is below $0.1 \mathrm{~s}$. The resulting multiaperture bistatic SAR image (Fig. 6(a)) is focused on the same grid as the single-aperture image shown in Fig. 4(a).

Fig. 6(b) and Fig. 6(c) present the single-aperture bistatic image, and respectively, the multi-aperture bistatic image of a high-rise building overlaid on a Google Earth picture. Based on the main response of the building, the measured $-6 \mathrm{~dB}$ resolution for the single-aperture image is $34.2 \mathrm{~m}$, while for the multi-aperture one is $6.8 \mathrm{~m}$.

Besides the drawback of a few focusing artifacts (that yield a measured peak to sidelobe ratio (PSLR) of around $26 \mathrm{~dB}$ ), in the multi-aperture image the scattering centers are much more clearly highlighted than in the single-aperture case. 


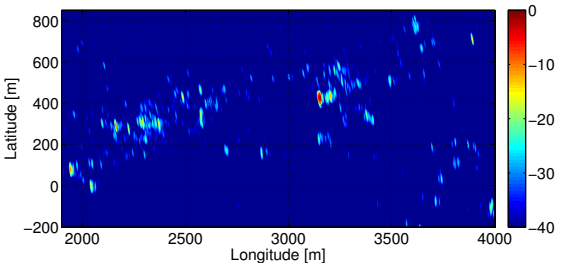

(a)

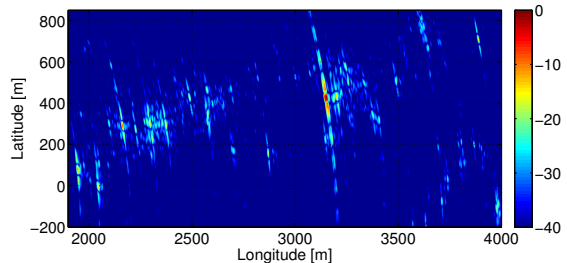

(b)

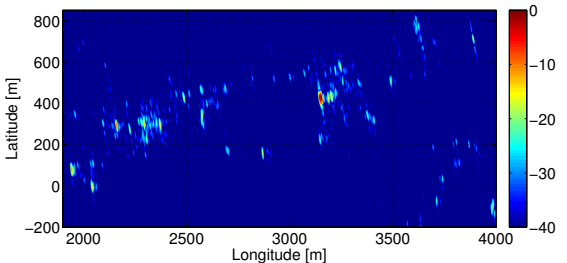

(c)

Fig. 4. Azimuth signal reconstruction assessment in multi-aperture focusing for an artificially introduced gap of $50 \mathrm{~ms}$ : (a) reference image, (b) focused image with the gap filled with zeros and (c) focused image with AR model-based filled gap.

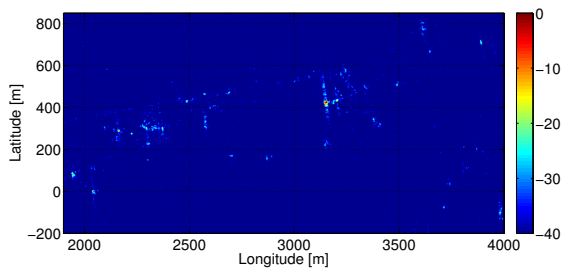

(a)

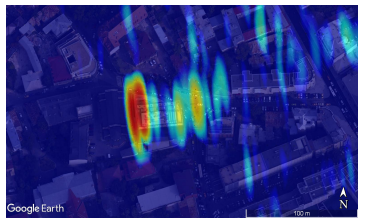

(b)

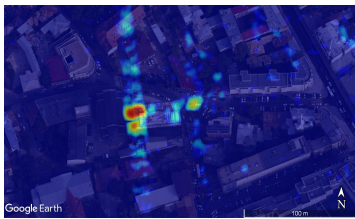

(c)
Fig. 6. Resolution enhancement with multi-aperture focusing: a) high-resolution multi-aperture focused bistatic SAR image; single-aperture (b) and multi-aperture (c) images of a highrise building overlaid on Google Earth.

\section{CONCLUSIONS}

This paper presented a methodology to focus spaceborne transmitter-stationary receiver bistatic data that span several along-track apertures with gaps in between. The possibility to increase the azimuth resolution using the proposed procedure was shown on a dataset acquired with Sentinel-1B operating in TOPSAR mode as transmitter of opportunity. The method can be further developed and future work will include: validation on scenes with richer information content, detailed characterization of the AR model-based bistatic azimuth signal reconstruction performances and evaluation of other signal reconstruction techniques (e.g., in [9] neural networks replace the AR model for audio inpainting).

\section{ACKNOWLEDGMENT}

This work has been funded by the European Space Agency (ESA) through the TomoSAR-1B project, contract number
4000124573/18/NL/CBi.

\section{REFERENCES}

[1] F. Behner, S. Reuter, H. Nies, and O. Loffeld, "Synchronization and Processing in the HITCHHIKER Bistatic SAR Experiment," IEEE J. Sel. Topics Appl. Earth Observ. Remote Sens., vol. 9, no. 3, pp. 1028-1035, 2016.

[2] S. Duque et al., "Repeat-pass interferometry using a fixed-receiver and ERS-2/ENVISAT as transmitters of opportunity," in IEEE IGARSS, Cape Town, South Africa, July 2009, vol. 2, pp. II-246-II-249.

[3] X. Fan et al., "Passive SAR with GNSS transmitters: Latest results and research progress," in IEEE IGARSS, July 2017, pp. 1043-1046.

[4] V. Kubica, X. Neyt, and H. D. Griffiths, "Along-track resolution enhancement for bistatic SAR imaging in burstmode operation," IEEE Trans. Aerosp. Electron. Syst., vol. 52, no. 4, pp. 1568-1575, Aug 2016.

[5] A. Anghel et al., "Bistatic SAR imaging with Sentinel-1 operating in TOPSAR mode," in IEEE Radar Conference, Seattle, WA, USA, May 2017, pp. 601-605.

[6] W. Etter, "Restoration of a discrete-time signal segment by interpolation based on the left-sided and right-sided autoregressive parameters," IEEE Transactions on Signal Processing, vol. 44, no. 5, pp. 1124-1135, May 1996.

[7] I. Kauppinen and K. Roth, "Audio Signal Extrapolation - Theory and Applications," in Proc. of the 5th Int. Conference on Digital Audio Effects (DAFx-02), Hamburg, Germany, September 2002, pp. 105-110.

[8] H. Akaike, "Fitting autoregressive models for prediction," Ann. Inst. Stat. Math., vol. 21, pp. 243-247, 1969.

[9] A. Marafioti, N. Perraudin, N. Holighaus, and P. Majdak, "A context encoder for audio inpainting," CoRR, vol. abs/1810.12138, 2018. 